\title{
Tensor products of hermitian lattices
}

\author{
by \\ Renaud Coulangeon (Talence)
}

1. Introduction. The properties of euclidean lattices with respect to tensor product have been studied in a series of papers by Kitaoka ([K, Chapter 7], [K1]). A rather natural problem which was investigated there, among others, was the determination of the short vectors in the tensor product $L \otimes M$ of two euclidean lattices $L$ and $M$. It was shown for instance that up to dimension 43 these short vectors are split, as one might hope.

The present paper deals with a similar question for tensor products of hermitian lattices over imaginary quadratic fields or quaternion division algebras. The main motivation for this work is in connection with modular lattices, as defined by Quebbemann $([\mathrm{Q}])$, that is to say, even lattices that are similar to their dual. In [B-N] it is shown how tensor product over the ring of integers in an imaginary quadratic field can be used to shift from one level to another (the level of a modular lattice $L$ is the square of the rate of the similarity mapping $L^{*}$ to $L$ ), and above all a construction of an 80dimensional extremal unimodular lattice from a 20-dimensional 7-modular one by tensoring is given. It is thus of some interest to know a priori how short vectors behave under tensor product.

In Section 2 we give the basic definitions and properties concerning hermitian lattices that are needed in the sequel. We establish in Section 3 a splitness criterion for minimal vectors (Corollary 3.4) based on a general lower bound (Proposition 3.2). Finally, Section 4 is devoted to examples; among others, we give an alternate proof of the extremality of Bachoc-Nebe's 80dimensional lattice, and we give a new construction of the Barnes-Wall lattices.

\section{Basic definitions}

2.1. Imaginary quadratic fields. Let $d$ be a square-free positive integer, $K=\mathbb{Q}(\sqrt{-d})$ and $\mathcal{O}_{K}$ the ring of integers in $K$. The complex conjugation,

2000 Mathematics Subject Classification: 11H55, 15A69. 
which induces the non-trivial element in $\operatorname{Gal}(K / \mathbb{Q})$, is denoted by ${ }^{-}$. Denote by $(V, h)$ an $m$-dimensional $K$-vector space $V$, endowed with a positive definite hermitian form $h$; an $\mathcal{O}_{K}$-submodule $L$ of $V$ is called an $\mathcal{O}_{K}$-lattice if it is finitely generated and contains a $K$-basis of $V$.

Since $h$ is positive definite, the "norm" function $x \mapsto h(x, x)$ admits a minimum on such a lattice $L$, denoted by $\mathrm{N}_{h}(L)$, which is attained on a finite set $S_{h}(L)$.

One defines the hermitian dual of $L$ as

$$
L^{\#}=\left\{y \in V \mid h(y, L) \subset \mathcal{O}_{K}\right\} .
$$

Given two $\mathcal{O}_{K}$-lattices $M \subset L$ in $(V, h)$, it is well known (Invariant Factor Theorem) that there exists a basis $\left\{e_{i}\right\}$ of $V$ and fractional ideals $\mathfrak{a}_{i}, \mathfrak{b}_{i}$ in $K$ such that $\mathfrak{b}_{i} \subset \mathfrak{a}_{i}$ and

$$
L=\mathfrak{a}_{1} e_{1} \oplus \ldots \oplus \mathfrak{a}_{m} e_{m}, \quad M=\mathfrak{b}_{1} e_{1} \oplus \ldots \oplus \mathfrak{b}_{m} e_{m} .
$$

This enables us to define the generalized index of $M$ in $L$ as the ideal

$$
\chi(L, M)=\prod_{i} \mathfrak{a}_{i}^{-1} \mathfrak{b}_{i} .
$$

In the more general case of two $\mathcal{O}_{K}$-lattices $L$ and $M$ in $(V, h)$ (with no inclusion), one gets a well defined generalized index setting

$$
\chi(L, M)=\chi(L, P) \chi(M, P)^{-1},
$$

where $P$ is any $\mathcal{O}_{K}$-sublattice of $L \cap M$ (for instance $P=L \cap M$ ).

The discriminant of $L$ is defined as $\delta_{L}=\chi\left(L^{\#}, L\right)$. The following proposition is rather well known (see [H, Proposition 2.3]).

Proposition 2.1.1. We have:

(1) $\delta_{L}=\operatorname{det}\left(h\left(e_{i}, e_{j}\right)\right) \prod_{i=1}^{m} \mathfrak{a}_{i} \overline{\mathfrak{a}}_{i}$.

(2) $\mathrm{N}_{K / \mathbb{Q}}(\chi(L, M))=\delta_{M} \delta_{L}^{-1}$.

Clearly, from the above proposition, $\delta_{L}$ is a principal ideal of $K$ admitting a unique non-negative generator in $\mathbb{Q}$ which we denote by $d_{L}$.

An $\mathcal{O}_{K}$-lattice of rank $m$ can of course be considered as a $\mathbb{Z}$-lattice of rank $2 m$, by setting

$$
x \cdot y=\operatorname{Tr}_{K / \mathbb{Q}}(h(x, y)) .
$$

With that convention, the norm $x \cdot x$ of a vector $x$ is twice its hermitian norm $h(x, x)$. The dual $L^{*}$ of $L$ with respect to that inner product is linked to $L^{\#}$ by

$$
L^{*}=\mathcal{D}_{K / \mathbb{Q}}^{-1} L^{\#},
$$

where $\mathcal{D}_{K / \mathbb{Q}}$ denotes the different of $K / \mathbb{Q}$, whence the relation

$$
\operatorname{det} L=\left|\mathfrak{d}_{K}\right|^{m}\left(d_{L}\right)^{2},
$$


in which $\mathfrak{d}_{K}$ denotes the discriminant of $K / \mathbb{Q}$. Let us finally define the invariant $\gamma_{h}(L)=\mathrm{N}_{h}(L) / d_{L}^{1 / m}$, which is related to the usual Hermite number $\gamma(L)$ by $\gamma_{h}(L)=\left(\sqrt{\mathfrak{d}_{K}} / 2\right) \gamma(L)$, and the constant $\gamma_{h, m}=\sup \left\{\gamma_{h}(L) \mid L \subset V\right\}$.

2.2. Quaternion fields. Much of the above definitions can be extended to the case of a quaternion field $H$ over $\mathbb{Q}$. As before, the conjugation in $H$ is denoted by ${ }^{-} ; \operatorname{Tr}_{H / \mathbb{Q}}$ (resp. tr) denotes the trace form of $H / \mathbb{Q}$ (resp. the reduced trace of $H / \mathbb{Q}$ ), $\mathrm{N}_{H / \mathbb{Q}}$ (resp. nr) denotes the norm of $H / \mathbb{Q}$ (resp. the reduced norm of $H / \mathbb{Q}$ ). We now fix a maximal order $\mathfrak{M}$ in $H$.

Let $(V, h)$ denote an $m$-dimensional left vector space over $H$ endowed with a positive definite hermitian form $h$. By a left $\mathfrak{M}$-lattice in $V$, we mean a finitely generated left $\mathfrak{M}$-module in $V$ containing an $H$-basis of $V$.

Definition (1) for the hermitian dual still holds, with $\mathcal{O}_{K}$ replaced by $\mathfrak{M}$. Next, any $\mathfrak{M}$-lattice $L$ can be written as a direct sum

$$
L=\mathfrak{a}_{1} e_{1} \oplus \ldots \oplus \mathfrak{a}_{m} e_{m},
$$

where $\mathfrak{a}_{i}$ are left $\mathfrak{M}$-ideals in $H$ (the only property of $\mathfrak{M}$ which is needed for this property to hold is heredity, which is fulfilled since $\mathfrak{M}$ is maximal, see [R, Theorem 2.44]).

In the non-commutative case, the definition of the index $\chi(L, M)$ has to be slightly modified. First we assume that $M \subset L$, and that the quotient $L / M$ is finite. The $\mathfrak{M}$-module $L / M$ is thus of finite length and admits a composition series

$$
L / M=L_{1} \supset \ldots \supset L_{r}
$$

in which each composition factor $L_{i} / L_{i+1}$ is isomorphic to $\mathfrak{M} / M_{i}$, where $M_{i}$ is a maximal left $\mathfrak{M}$-ideal. By the Jordan-Hölder theorem, the set $\left\{M_{i}\right\}$ is uniquely determined up to isomorphism by $L / M$, thus we get a well defined index setting

$$
\chi(L, M)=\prod \operatorname{nr}\left(M_{i}\right) .
$$

In the general case, we set

$$
\chi(L, M)=\chi(L, L \cap M) \chi(M, L \cap M)^{-1}=\chi(L, P) \chi(M, P)^{-1},
$$

for any $\mathfrak{M}$-sublattice $P$ of $L \cap M$. The following lemma suggests an appropriate definition of the discriminant of $L$ :

LEMMA 2.2.1. Let $L=\mathfrak{a}_{1} e_{1} \oplus \ldots \oplus \mathfrak{a}_{m} e_{m}$ be an $\mathfrak{M}$-lattice in a left hermitian space $(V, h)$. Then

$$
\chi\left(L^{\#}, L\right)=\operatorname{nr}\left(h\left(e_{i}, e_{j}\right)\right) \prod_{i=1}^{m} \operatorname{nr}\left(\mathfrak{a}_{i}\right)^{2}=d^{2} \mathbb{Z},
$$

where $d$ is a positive element in $\mathbb{Q}$. 
Proof. Let $\left\{e_{i}^{\#}\right\}$ be the dual basis of $\left\{e_{i}\right\}$ (i.e. $\left.h\left(e_{i}^{\#}, e_{j}\right)=\delta_{i j}\right)$. The hermitian dual $L^{\#}$ of $L$ satisfies $L^{\#}=\overline{\mathfrak{a}}_{1}^{-1} e_{1}^{\#} \oplus \ldots \oplus \overline{\mathfrak{a}}_{n}^{-1} e_{n}^{\#}$. It suffices to establish the formula

$$
\chi\left(L^{\#}, L\right)=\operatorname{nr}\left(h\left(e_{i}, e_{j}\right)\right) \prod_{i=1}^{m} \operatorname{nr}\left(\mathfrak{a}_{i}\right)^{2},
$$

after localizing at an arbitrary prime $p$. Since $\mathfrak{M}_{p}$ is (left) principal for any $p$, this reduces to a standard calculation, just as in the usual case, i.e. lattices over Dedekind rings (cf. for instance [Se, chapitre III]).

For the second equality of the lemma, we only need to check that $\operatorname{nr}\left(h\left(e_{i}, e_{j}\right)\right)$ is a square. Since $h$ is positive definite, there exists a matrix $P \in \mathcal{M}_{m}(H)$ and a diagonal matrix $D$ with rational positive entries $d_{1}, \ldots, d_{m}$ such that

$$
\left(h\left(e_{i}, e_{j}\right)\right)={ }^{t} \bar{P} D P .
$$

Let $E$ be a splitting field of $H$, i.e. $E \otimes_{\mathbb{Q}} H \simeq \mathcal{M}_{2}(E)$. By the definition of the reduced norm, we have

$$
\operatorname{nr}\left(h\left(e_{i}, e_{j}\right)\right)=\operatorname{det}\left(1 \otimes{ }^{t} \bar{P}\right) \operatorname{det}(1 \otimes D) \operatorname{det}(1 \otimes P),
$$

where all the determinants are calculated in $E \otimes_{\mathbb{Q}} \mathcal{M}_{m}(H) \simeq \mathcal{M}_{2 m}(E)$. It is then easily checked that $\overline{{ }^{t}(1 \otimes P)}=1 \otimes{ }^{t} \bar{P}$, whence $\operatorname{nr}\left({ }^{t} \bar{P}\right)=\overline{\operatorname{nr} P}=\operatorname{nr} P$, since $\operatorname{nr} P \in \mathbb{Q}$. Consequently, $\operatorname{nr}\left(h\left(e_{i}, e_{j}\right)\right)=\left(\operatorname{nr} P \prod_{i=1}^{m} d_{i}\right)^{2}$.

We thus define the discriminant $\delta_{L}$ of $L$ as $\sqrt{\chi\left(L^{\#}, L\right)}$, and denote by $d_{L}$ its unique positive generator in $\mathbb{Q}$. With that convention, we immediately obtain:

Proposition 2.2.2. $\chi(L, M)=(\delta M)(\delta L)^{-1}$.

As before, we make $L$ into an ordinary $4 m$-dimensional $\mathbb{Z}$-lattice, setting $x \cdot y=\operatorname{tr}(h(x, y))$. Thus $x \cdot x=2 h(x, x)$ and the norm of $L$ is twice its hermitian norm $\mathrm{N}_{h}(L)$. The dual $L^{*}$ of $L$ with respect to this inner product is linked to $\Lambda^{\#}$ by

$$
L^{*}=\mathcal{D}_{H / \mathbb{Q}}^{-1} L^{\#},
$$

where $\mathcal{D}_{H / \mathbb{Q}}$ denotes the different of $H / \mathbb{Q}$. This yields the formula

$$
\operatorname{det} L=\mathfrak{d}_{H}^{m}\left(d_{L}\right)^{4},
$$

in which $\mathfrak{d}_{H}=\mathrm{N}_{H / \mathbb{Q}}\left(\mathcal{D}_{H / \mathbb{Q}}\right)$ is the discriminant of $H / \mathbb{Q}$ with respect to the reduced trace.

As before we define $\gamma_{h}(L)=\mathrm{N}_{h}(L) / d_{L}^{1 / m}$, which is now related to $\gamma(L)$ by $\gamma_{h}(L)=\left(\mathfrak{d}_{H}^{1 / 4} / 2\right) \gamma(L)$, and we set $\gamma_{h, m}=\sup \left\{\gamma_{h}(L) \mid L \subset V\right\}$. 
3. Tensor product of hermitian lattices. In the (commutative) case of imaginary quadratic fields, we define the tensor product $\left(V_{1} \otimes_{K} V_{2}, h_{1} \otimes h_{2}\right)$ of two hermitian spaces $\left(V_{1}, h_{1}\right)$ and $\left(V_{2}, h_{2}\right)$ in the usual way, setting

$$
h_{1} \otimes h_{2}\left(x_{1} \otimes x_{2}, y_{1} \otimes y_{2}\right)=h_{1}\left(x_{1}, y_{1}\right) h_{2}\left(x_{2}, y_{2}\right)
$$

for split elements, which is easily seen to extend to a well defined hermitian form on the underlying space $V_{1} \otimes_{K} V_{2}$. Notice, for instance taking orthogonal bases, that $h_{1} \otimes h_{2}$ is positive definite if $h_{1}$ and $h_{2}$ are.

In the quaternionic case, we cannot define the "tensor product" of two hermitian forms. We can nevertheless overcome this difficulty in the following way: let $\left(V_{1}, h_{1}\right)$ be a right $H$-vector space endowed with a right sesquilinear form $h_{1}$, i.e.

$$
\begin{aligned}
& h_{1}(x \alpha+y \beta, z)=h_{1}(x, z) \alpha+h_{1}(y, z) \beta, \\
& h_{1}(x, y \alpha+z \beta)=\bar{\alpha} h_{1}(x, y)+\bar{\beta} h_{1}(x, z)
\end{aligned}
$$

(e.g. $V_{1}=H$ viewed as a right $H$-module and $h_{1}(x, y)=\bar{y} x$ ). Alternatively, let $\left(V_{2}, h_{2}\right)$ be a left $H$-vector space endowed with a left sesquilinear form $h_{2}$, i.e.

$$
\begin{aligned}
& h_{2}(\alpha x+\beta y, z)=\alpha h_{2}(x, z)+\beta h_{2}(y, z), \\
& h_{2}(x, \alpha y+\beta z)=h_{2}(x, y) \bar{\alpha}+h_{2}(x, z) \bar{\beta}
\end{aligned}
$$

(e.g. $V_{2}=H$ viewed as a left $H$-module and $h_{2}(x, y)=x \bar{y}$ ).

The tensor product $V_{1} \otimes_{H} V_{2}$ is then well defined (see [Bou, chap. II, $\S 3])$. Although there is no well defined sesquilinear form $h_{1} \otimes h_{2}$ on $V_{1} \otimes_{H} V_{2}$ satisfying $h_{1} \otimes h_{2}(x \otimes y, z \otimes t)=h_{1}(x, z) h_{2}(y, t)$ (conditions of the type $h_{1} \otimes h_{2}(x \otimes y, z a \otimes t)=h_{1} \otimes h_{2}(x \otimes y, z \otimes a t)$ cannot be satisfied unless $H$ is commutative), we get nevertheless a well defined $\mathbb{Q}$-bilinear form $f$ on $V_{1} \otimes_{H} V_{2}$ by taking the trace of the above formula:

$$
f(x \otimes y, z \otimes t):=\operatorname{tr}\left(h_{1}(x, z) h_{2}(y, t)\right) .
$$

Moreover, if $h_{1}$ and $h_{2}$ are hermitian then $f$ is symmetric, and it is positive definite if $h_{1}$ and $h_{2}$ are. One can also define the hermitian norm of an element $\omega=\sum_{i=1}^{r} x_{i} \otimes y_{i}$ in $V_{1} \otimes_{H} V_{2}$ by

$$
h(\omega, \omega):=\sum_{i} h_{1}\left(x_{i}, x_{i}\right) h_{2}\left(y_{i}, y_{i}\right)+\operatorname{tr} \sum_{i<j} h_{1}\left(x_{i}, x_{j}\right) h_{2}\left(y_{i}, y_{j}\right),
$$

which is well defined and linked to $f$ by $f(\omega, \omega)=2 h(\omega, \omega)$.

We try from now on to state the results for the quadratic and the quaternionic cases in a uniform way, setting $K$ for an imaginary quadratic field or a quaternion field, and $\mathcal{O}_{K}$ for a maximal order in $K$ (of course unique in the quadratic case).

Our basic task is, given two $\mathcal{O}_{K}$-lattices $L$ and $M$, to determine the minimal norm of $L \otimes_{\mathcal{O}_{K}} M$, and the set $S_{h}\left(L \otimes_{\mathcal{O}_{K}} M\right)$ on which it is attained. 
We introduce some more notation, setting

$$
\delta_{k} L:=\min \left\{\delta_{M} \mid M \text { free } \mathcal{O}_{K} \text {-sublattice of } L, \operatorname{rank}_{\mathcal{O}_{K}} M=k\right\},
$$

and $d_{k} L$ for its positive generator. We first prove the following lemma:

Lemma 3.1. Let $\omega=\sum_{i=1}^{r} x_{i} \otimes y_{i}$ be a vector in $L \otimes_{\mathcal{O}_{K}} M$, and assume that $r$ is the minimal length of such a decomposition for $\omega$ (we call $r$ the rank of $\omega)$. Then the $x_{i}$ (resp. $\left.y_{i}\right)$ generate a submodule of rank $r$ of $L$ (resp. $\left.M\right)$. Incidentally, $r \leq \min \{\operatorname{rank} L, \operatorname{rank} M\}$.

Proof. To handle simultaneously the quadratic and quaternionic cases, we have to assume that $L$ and $M$ are respectively right and left $\mathcal{O}_{K}$-modules. Let $t$ be the rank of the (right) $\mathcal{O}_{K}$-module $\widetilde{L}$ generated by $x_{1}, \ldots, x_{r}$. Using (2) or (7), one can write

$$
\widetilde{L}=e_{1} \mathfrak{a}_{1} \oplus \ldots \oplus e_{t} \mathfrak{a}_{t},
$$

where $\mathfrak{a}_{i}$ are (right) fractional ideals of $K$. Set $x_{i}=\sum_{j=1}^{t} e_{j} a_{i, j}, a_{i, j} \in \mathfrak{a}_{j}$. If $t<r$, we obtain $\omega=\sum_{j=1}^{t} e_{j} \otimes\left(\sum_{i=1}^{r} a_{i, j} y_{i}\right)$, which contradicts the minimality of $r$.

Proposition 3.2. Let $\omega$ be a minimal vector in $L \otimes_{\mathcal{O}_{K}} M$ of rank $r$. Then its hermitian norm $h(\omega, \omega)$ satisfies

$$
h(\omega, \omega) \geq r\left(d_{r} L\right)^{1 / r}\left(d_{r} M\right)^{1 / r} .
$$

The proof is similar to Kitaoka's proof for tensor product over $\mathbb{Z}[\mathrm{K}$, Chapter 7], and is based on the following lemma:

Lemma 3.3. Let $A$ and $B$ be two positive definite hermitian matrices in $\mathcal{M}_{r}(K)$, where $K$ is either an imaginary quadratic field or a quaternion field.

(1) If $K$ is an imaginary quadratic field, then

$$
\operatorname{Tr} A B \geq r(\operatorname{det} A)^{1 / r}(\operatorname{det} B)^{1 / r} .
$$

(2) If $K$ is a quaternion field, then

$$
\operatorname{tr}(\operatorname{Tr} A B) \geq 2 r(\mathrm{nr} A)^{1 /(2 r)}(\mathrm{nr} B)^{1 /(2 r)} .
$$

Proof. In the quadratic imaginary case, this is a straightforward adaptation of the well known analogous statement for positive definite symmetric matrices (see [K, Lemma 7.1.3]). The quaternionic case reduces to the previous case in the following way: one can find an imaginary quadratic field $E$ which is a splitting field for $K$. Then

$$
\operatorname{tr}\left(\operatorname{Tr}_{\mathcal{M}_{r}(K) / K} A B\right)=\operatorname{Tr}_{\mathcal{M}_{2 r}(E) / E}((1 \otimes A)(1 \otimes B))
$$

where $1 \otimes A$ and $1 \otimes B$ denote the images of $A$ and $B$ in $\mathcal{M}_{2 r}(E)$, and $\operatorname{tr}$ denotes the reduced trace of $K / \mathbb{Q}$. It is easily checked that $1 \otimes A$ is hermitian, 
resp. positive definite hermitian, if $A$ already is, so that we can apply the first part of the lemma to (10) and obtain

$$
\begin{aligned}
\operatorname{Tr}_{\mathcal{M}_{2 r}}(E) / E((1 \otimes A)(1 \otimes B)) & \geq 2 r(\operatorname{det}(1 \otimes A))^{1 /(2 r)}(\operatorname{det}(1 \otimes B))^{1 /(2 r)} \\
& =2 r(\operatorname{nr} A)^{1 /(2 r)}(\mathrm{nr} B)^{1 /(2 r)} .
\end{aligned}
$$

Proof of 3.2. Setting $A=\left(h\left(x_{i}, x_{j}\right)\right)_{1 \leq i, j \leq r}, B=\left(h\left(y_{i}, y_{j}\right)\right)_{1 \leq i, j \leq r}$, we see that $h(\omega, \omega)=\sum_{i, j} h\left(x_{i}, x_{j}\right) h\left(y_{i}, y_{j}\right)=\operatorname{Tr} A \bar{B} \geq r(\operatorname{det} A)^{1 / r}(\operatorname{det} \bar{B})^{1 / r}$ in the imaginary quadratic case, and $h(\omega, \omega)=\frac{1}{2} f(\omega, \omega)=\operatorname{tr}(\operatorname{Tr} A \bar{B}) \geq$ $r(\mathrm{nr} A)^{1 /(2 r)}(\mathrm{nr} \bar{B})^{1 /(2 r)}$ in the quaternionic case. In both cases, the righthand side of the inequality is equal to $r\left(d_{\widetilde{L}}\right)^{1 / r}\left(d_{\widetilde{M}}\right)^{1 / r}$, where $\widetilde{L}$ (resp. $\widetilde{M}$ ), denotes the submodule generated by the $x_{i}\left(\operatorname{resp} . y_{i}\right)$, whence the conclusion upon taking lower bounds. then

Corollary 3.4. Set $r_{K}=\sup \left\{r>1 \mid \gamma_{h, r}^{2} / r<1\right\}$. If $\operatorname{rank} L \leq r_{K}$,

$$
S_{h}\left(L \otimes \mathcal{O}_{K} M\right)=\left\{x \otimes y \mid x \in S_{h}(L), y \in S_{h}(M)\right\},
$$

for any positive definite hermitian lattice $M$.

Proof. Since $h(\omega, \omega)$ is naturally bounded above by $\mathrm{N}_{h}(L) \mathrm{N}_{h}(M)$, the inequality of Proposition 3.2 implies

$$
\gamma_{h}(\widetilde{L}) \gamma_{h}(\widetilde{M})=\frac{\mathrm{N}_{h}(\widetilde{L})}{\left(d_{\widetilde{L}}\right)^{1 / r}} \cdot \frac{\mathrm{N}_{h}(\widetilde{M})}{\left(d_{\widetilde{M}}\right)^{1 / r}} \geq r,
$$

whence $\gamma_{h, r}^{2} \geq r$, which, from the definition of $r_{K}$, implies that $r=1$, completing the proof.

EXAMPLE. Using the known values of $\gamma_{n}$, for $n=1, \ldots, 8$, and the upper bound $\gamma_{n} \leq(4 / \pi) \Gamma(1+n / 2)^{2 / n}$ for $n \geq 9$, one can check that $r_{\mathbb{Q}(\sqrt{-3})} \geq 4$.

\section{Examples}

4.1. $K=\mathbb{Q}(i)$. Let $\Lambda_{24}$ be the Leech lattice, viewed as a hermitian $\mathbb{Z}[i]$-lattice. Denote by $\mathfrak{M}_{0}$ the Hurwitz order in $\mathbb{Q}_{2, \infty}$, which, together with the inner product $x \cdot y=\operatorname{tr}(x \bar{y})$ (reduced trace), is well known to be isometric to $\mathbb{D}_{4}$ (see for instance [M, p. 225]). As $\mathbb{Z}[i]$ embeds in $\mathfrak{M}_{0}$ (explicitly $\mathfrak{M}_{0}=$ $\mathbb{Z}[i] \oplus \mathbb{Z}[i] \omega$, where $\omega=(-1+i+j+k) / 2)$, any $\mathfrak{M}_{0}$-lattice of rank $m$ can be viewed as a $\mathbb{Z}[i]$-lattice of rank $2 m$, with hermitian form

$$
h=\left(\begin{array}{cc}
1 & \frac{-1+i}{2} \\
\frac{-1-i}{2} & 1
\end{array}\right) \otimes I_{m} .
$$

Set $\mathfrak{P}=(1+i) \mathfrak{M}_{0}=\mathfrak{M}_{0}(1+i)$. As a $\mathbb{Z}[i]$-lattice it satisfies $\mathfrak{P}^{\#}=\frac{1-i}{2} \mathfrak{P}$.

Proposition 4.1.1. $\mathfrak{P} \otimes_{\mathbb{Z}[i]} \Lambda_{24}$ is an extremal 48-dimensional 2-modular lattice. 
Proof. We have $\Lambda_{24}^{\#}=2 i \Lambda_{24}^{*}=2 i \Lambda_{24}$, and $\mathfrak{P}^{\#}=\frac{1-i}{2} \mathfrak{P}$, so that

$$
\left(\mathfrak{P} \otimes \Lambda_{24}\right)^{*}=\frac{1}{2 i}\left(\mathfrak{P} \otimes \Lambda_{24}\right)^{\#}=\frac{1}{2 i} \mathfrak{P}^{\#} \otimes \Lambda_{24}^{\#}=\frac{1-i}{2} \mathfrak{P} \otimes \Lambda_{24},
$$

whence the 2-modularity. We have to check that $\mathrm{N}_{h}\left(\mathfrak{P} \otimes \Lambda_{24}\right) \geq 8$, that is, $\mathrm{N}_{h}\left(\mathfrak{P} \otimes \Lambda_{24}\right) \geq 4$. Any minimal vector of $\mathfrak{P} \otimes \Lambda_{24}$ is of the shape $\omega=$ $\sum_{i=1}^{r} x_{i} \otimes y_{i}$, with $r \leq 2$. Since $\mathrm{N}_{h}\left(\Lambda_{24}\right)=\mathrm{N}_{h}(\mathfrak{P})=2$, we are done with the split case $r=1$. In the case $r=2$, we have, from Proposition 3.2,

$$
h(\omega, \omega) \geq 2\left(d_{2}(\mathfrak{P})\right)^{1 / 2}\left(d_{2}\left(\Lambda_{24}\right)\right)^{1 / 2}=2 \sqrt{2}\left(d_{2}\left(\Lambda_{24}\right)\right)^{1 / 2} .
$$

So we only need to check that $d_{2}\left(\Lambda_{24}\right) \geq 2$, which is clear since

$$
\frac{2}{\left(d_{2}\left(\Lambda_{24}\right)\right)^{1 / 2}} \leq \gamma_{4}=\sqrt{2}
$$

REMARK. I owe to the referee the remark that in $[\mathrm{B}]$, a construction, due to H. G. Quebbemann, of an extremal 2-modular lattice in dimension 48 is mentioned (see Theorem 6.7 of [B] and its proof), which turns out to be equivalent to ours.

4.2. $K=\mathbb{Q}(\sqrt{-7})$. In [ATLAS, p. 39], a hermitian unimodular 20dimensional lattice $L_{20}$ over $\mathcal{O}_{K}=\mathbb{Z}[\alpha](\alpha=(1+\sqrt{-7}) / 2)$, with (hermitian) minimal norm 4 is explicitly given. As a $\mathbb{Z}$-lattice, $L_{20}$ is thus 7 -modular of norm 8 (extremal). From this, C. Bachoc and G. Nebe constructed in [B-N] a 40-dimensional 3-modular lattice $L_{40}$, and an 80-dimensional unimodular $L_{80}$, and showed that they are both extremal, i.e. of norm 8 . Their construction is as follows: they first notice that the lattices $\mathbb{A}_{2}^{2}$ (orthogonal sum of two copies of $\mathbb{A}_{2}$ ) and $\mathbb{E}_{8}$ admit a hermitian structure over $\mathcal{O}_{K}$, with the following hermitian Gram matrices:

$$
a_{2}^{2}=\left(\begin{array}{cc}
1 & \frac{2}{\sqrt{-7}} \\
\frac{-2}{\sqrt{-7}} & 1
\end{array}\right) \quad \text { and } \quad e_{8}=\left(\begin{array}{cccc}
1 & \frac{2}{\sqrt{-7}} & \frac{-\alpha}{\sqrt{-7}} & 0 \\
\frac{-2}{\sqrt{-7}} & 1 & 0 & \frac{-\alpha}{\sqrt{-7}} \\
\frac{\bar{\alpha}}{\sqrt{-7}} & 0 & 1 & \frac{-2}{\sqrt{-7}} \\
0 & \frac{\alpha}{\sqrt{-7}} & \frac{2}{\sqrt{-7}} & 1
\end{array}\right)
$$

Then they define

$$
L_{40}:=\mathbb{A}_{2}^{2} \otimes_{\mathcal{O}_{K}} L_{20} \quad \text { and } \quad L_{80}:=\mathbb{E}_{8} \otimes_{\mathcal{O}_{K}} L_{20} .
$$

TheOREM 4.2.1 (Bachoc \& Nebe). $L_{80}$ (resp. $\left.L_{40}\right)$ is unimodular (resp. 3-modular) of minimum 8.

Proof. For the assertion concerning modularity, we refer to [B-N] (the proof is similar to that of Proposition 4.1.1 above). So we only have to show that $\mathrm{N}_{h}\left(L_{40}\right)$ and $\mathrm{N}_{h}\left(L_{80}\right)$ are $>3$, which by Proposition 3.2, amounts to estimating $d_{r}\left(L_{20}\right)$ for $r \leq 4$. 
LEMMA 4.2.2. We have:

(1) $d_{2}\left(L_{20}\right)=8$.

(2) $d_{3}\left(L_{20}\right)=8$.

Proof. (1) Let $M$ be a (free) $\mathcal{O}_{K}$-lattice of rank 2. Taking a vector $x$ with minimal hermitian norm $h(x, x)$ as first element of an $\mathcal{O}_{K}$-basis $\{x, y\}$ of $M$, which is possible since $\mathrm{Cl}_{K}=1$, we see that

$$
d_{M}=h(x, x) h(y-p(y), y-p(y)),
$$

where $p$ denotes the orthogonal projection onto $K x$. Set $p(y)=\beta x$, with $\beta \in K$. Since $\mathcal{O}_{K}$ is euclidean with respect to the norm, with euclidean minimum $4 / 7$, we can assume, replacing $y$ by $y+u x$ if necessary, with a suitable algebraic integer $u$ of $\mathcal{O}_{K}$, that $\beta \bar{\beta} \leq 4 / 7$. Consequently,

$$
d_{M} \geq h(x, x)\left(h(y, y)-\frac{4}{7} h(x, x)\right) \geq \frac{3}{7} h(x, x) h(y, y),
$$

so that

$$
d_{2}\left(L_{20}\right) \geq \frac{3}{7} \mathrm{~N}_{h}\left(L_{20}\right)^{2}>6 .
$$

Then, if $M$ is a 2-dimensional $\mathcal{O}_{K}$-section of $L_{20}$ with $d_{M} \leq 8$, it admits a basis $\{x, y\}$ satisfying

$$
16 \leq h(x, x) h(y, y) \leq 56 / 3<19,
$$

whence $h(x, x)=h(y, y)=4$, and $d_{M}=16-\mathrm{N}_{K / \mathbb{Q}}(h(x, y))$. Finally, we cannot have $d_{M}=7$, since it would imply $h(x, y)=3$, whence $h(x-y, x-y)$ $=2$, which contradicts $\mathrm{N}_{h}\left(L_{20}\right)=4$. Thus $d_{2}\left(L_{20}\right)=8$, and the above discussion shows that any minimal section $M_{2}$ admits an hermitian Gram matrix of the form

$$
\left(\begin{array}{cc}
4 & 2 \alpha \\
2 \bar{\alpha} & 4
\end{array}\right)
$$

(2) Let $M_{3}$ be a minimal 3-dimensional section of $L_{20}$, and suppose that $d_{3}\left(L_{20}\right) \leq 8$. We claim that $M_{3}$ contains a minimal 2-dimensional minimal section $M_{2}$. In fact, the well known relation

$$
d_{M}=\frac{d_{r}(M)}{d_{n-r}\left(M^{\#}\right)},
$$

valid for any $n$-dimensional $\mathcal{O}_{K}$-lattice $M$ and any $r \leq n$, together with the density inequality

$$
\frac{\mathrm{N}_{h}\left(M_{3}^{\#}\right)}{\left(d\left(M_{3}^{\#}\right)\right)^{1 / 3}} \leq \frac{\sqrt{7}}{2} \gamma_{6}=\frac{\sqrt{7}}{2}\left(\frac{64}{3}\right)^{1 / 6},
$$

leads to

$$
d_{2}\left(M_{3}\right) \leq 2 \sqrt{7}\left(\frac{64}{3}\right)^{1 / 6}<9
$$


whence $d_{2}\left(M_{3}\right)=8$ and $M_{3}$ contains a section $M_{2}$. Writing

$$
M_{3}=M_{2}+\mathcal{O}_{K} x
$$

and denoting by $p(x)$ the orthogonal projection of $x$ onto the subspace $K M_{2}$, and $q(x)$ its projection onto the orthogonal complement of $K M_{2}$, we have

$$
d_{M_{3}}=d_{M_{2}} h(q(x), q(x))=8(h(x, x)-h(p(x), p(x))) .
$$

Moreover, $p(x) \in M_{2}^{\#}$, and up to isometry, $M_{3}$ depends only on $h(x, x)$ and on the class of $p(x)$ in $M_{2}^{\#} / M_{2}$. Thus we have to select representatives for these classes. Let $\left\{e_{1}, e_{2}\right\}$ be a basis of $M_{2}$ with Gram matrix of type (*). It is readily checked that

whence

$$
M_{2}^{\#}=\mathcal{O}_{K} \frac{\bar{\alpha}}{4} e_{1} \oplus \mathcal{O}_{K} \frac{\alpha}{4} e_{2}
$$

$$
M_{2}^{\#} / M_{2} \simeq \mathcal{O}_{K} /(2 \alpha) \times \mathcal{O}_{K} /(2 \bar{\alpha}) .
$$

Thus, if we set $f_{1}=\frac{\bar{\alpha}}{4} e_{1}$ and $f_{2}=\frac{\alpha}{4} e_{2}$, then the elements $\varepsilon_{1} f_{1}+\bar{\varepsilon}_{2} f_{2}$, as $\varepsilon_{i}$ ranges over the set $\left\{0, \alpha, \alpha^{2},-2, \bar{\alpha}, 1,-1,-\bar{\alpha}\right\}$, provide a set of representatives of $M_{2}^{\#} / M_{2}$. The hermitian norms of these elements are summarized in the following array in which the rows (resp. columns) correspond to the coordinate $\varepsilon_{1}$ (resp. $\left.\bar{\varepsilon}_{2}\right)$.

\begin{tabular}{|c|cccccccc|}
\hline & 0 & $\bar{\alpha}$ & $\bar{\alpha}^{2}$ & -2 & $\alpha$ & 1 & -1 & $-\alpha$ \\
\hline 0 & 0 & 1 & 2 & 2 & 1 & $1 / 2$ & $1 / 2$ & 1 \\
$\alpha$ & 1 & $5 / 2$ & $3 / 2$ & 1 & $5 / 2$ & $5 / 2$ & $1 / 2$ & $3 / 2$ \\
$\alpha^{2}$ & 2 & $3 / 2$ & $3 / 2$ & 3 & 5 & 3 & 2 & 1 \\
-2 & 2 & 1 & 3 & 5 & $9 / 2$ & 2 & 3 & $3 / 2$ \\
$\bar{\alpha}$ & 1 & $5 / 2$ & 5 & $9 / 2$ & $3 / 4$ & $3 / 4$ & $9 / 4$ & $13 / 4$ \\
1 & $1 / 2$ & $5 / 2$ & 3 & 2 & $3 / 4$ & $5 / 4$ & $3 / 4$ & $9 / 4$ \\
-1 & $1 / 2$ & $1 / 2$ & 2 & 3 & $9 / 4$ & $3 / 4$ & $5 / 4$ & $3 / 4$ \\
$-\bar{\alpha}$ & 1 & $3 / 2$ & 1 & $3 / 2$ & $13 / 4$ & $9 / 4$ & $3 / 4$ & $3 / 4$ \\
\hline
\end{tabular}

Using (**), we see that if $h(p(x), p(x)) \in \mathbb{Z}$, then $h(q(x, x))$ is a positive integer so that $d_{M_{3}} \geq 8$. If $h(p(x), p(x)) \notin \mathbb{Z}$ but $h(p(x), p(x)) \leq 3$, then again $d_{M_{3}}=8(h(x, x)-h(p(x), p(x))) \geq 8$.

So the only remaining case is $p(x) \equiv \pm\left(\bar{\alpha} f_{1}-\alpha f_{2}\right) \bmod M_{2}$, which could a priori lead to $d_{M_{3}}=8(4-13 / 4)<8$. This would imply that $\bar{\alpha} f_{1}-\alpha f_{2}$ is the projection onto $K M_{2}$ of a vector $x$ of hermitian norm 4 . But then the discriminant of the 2 -dimensional $\mathcal{O}_{K}$-lattice spanned by $x$ and $e_{1}$ would be $h(x, x) h\left(e_{1}, e_{1}\right)-\mathrm{N}_{K / \mathbb{Q}}\left(h\left(x, e_{1}\right)\right)=5$, which is impossible since $d_{2}\left(L_{20}\right)=8$. This completes the proof that $d_{3}\left(L_{20}\right) \geq 8$. Further, this actually is an equality, since $L_{20}$ contains a section of discriminant 8 ([B-N]) (the inequality is, however, sufficient for our purpose). 
We are now able to prove Theorem 4.2.1. We first deal with $L_{40}$ : any minimal vector $\omega$ admits a decomposition of rank $r \leq 2$, and in the split case $r=1$, we have $h(\omega, \omega) \geq \mathrm{N}_{h}\left(\mathbb{A}_{2}^{2}\right) \mathrm{N}_{h}\left(L_{20}\right)=4$. If $r=2$, then Proposition 3.2 together with Lemma 4.2.2 implies that

$$
h(\omega, \omega) \geq 2\left(d_{\mathbb{A}_{2}}^{2}\right)^{1 / 2}\left(d_{2}\left(L_{20}\right)\right)^{1 / 2}=2 \sqrt{3 / 7} \sqrt{8}>3 .
$$

Consequently, $\mathrm{N}_{h}\left(L_{40}\right)=4$ and $L_{40}$ is extremal.

In the case of $L_{80}$, the same type of argument shows that any vector with a decomposition of rank $r=1,2,3$ has hermitian norm $>3$ : we need for this, in addition to Lemma 4.2.2, to know the actual value of $d_{r}\left(\mathbb{E}_{8}\right)$ for $r=1,2,3$, namely $\delta_{h, r}\left(\mathbb{E}_{8}\right)=1,3 / 7,1 / 7$ respectively, as one easily checks. Finally, we have to deal with the rank 4 case, which will be done by a direct computation, since $d_{4}\left(L_{20}\right)$ is not known. Let $\left\{b_{1}, \ldots, b_{4}\right\}$ be an $\mathcal{O}_{K}$-basis of $\mathbb{E}_{8}$, corresponding to a Gram matrix of the shape $e_{8}$. Any rank 4 element $\omega$ of $L_{80}=\mathbb{E}_{8} \otimes_{\mathcal{O}_{K}} L_{20}$ can be written in the form $\omega=\sum_{i=1}^{4} b_{i} \otimes x_{i}$, where $x_{i} \in L_{20}, i=1, \ldots, 4$. Then

$$
\begin{aligned}
h(\omega, \omega)= & \sum_{i=1}^{4} h\left(x_{i}, x_{i}\right)+\operatorname{Tr}_{K / \mathbb{Q}}\left(\frac{2}{\sqrt{-7}} h\left(x_{1}, x_{2}\right)\right)-\operatorname{Tr}_{K / \mathbb{Q}}\left(\frac{\alpha}{\sqrt{-7}} h\left(x_{1}, x_{3}\right)\right) \\
& -\operatorname{Tr}_{K / \mathbb{Q}}\left(\frac{\alpha}{\sqrt{-7}} h\left(x_{2}, x_{4}\right)\right)-\operatorname{Tr}_{K / \mathbb{Q}}\left(\frac{2}{\sqrt{-7}} h\left(x_{3}, x_{4}\right)\right) \\
= & \sum_{i=1}^{4} h\left(x_{i}, x_{i}\right)+\operatorname{Tr}_{K / \mathbb{Q}}\left(\frac{\alpha}{\sqrt{-7}} h\left(x_{1}, \alpha x_{2}-x_{3}\right)\right) \\
& -\operatorname{Tr}_{K / \mathbb{Q}}\left(\frac{\alpha}{\sqrt{-7}} h\left(\bar{\alpha} x_{3}+x_{2}, x_{4}\right)\right) .
\end{aligned}
$$

The last two terms on the right-hand side can be bounded above, namely

$$
\begin{aligned}
\left|\operatorname{Tr}_{K / \mathbb{Q}}\left(\frac{\alpha}{\sqrt{-7}} h\left(x_{1}, \alpha x_{2}-x_{3}\right)\right)\right| \leq & h\left(x_{1}, x_{1}\right)+\frac{2}{7} h\left(\alpha x_{2}-x_{3}, \alpha x_{2}-x_{3}\right) \\
& -\mathrm{N}_{h}\left(\mathcal{O}_{K} x_{1} \oplus \mathcal{O}_{K} \frac{\bar{\alpha}}{\sqrt{-7}}\left(\alpha x_{2}-x_{3}\right)\right) \\
\leq & h\left(x_{1}, x_{1}\right)+\frac{4}{7} h\left(x_{2}, x_{2}\right)+\frac{2}{7} h\left(x_{3}, x_{3}\right) \\
& -\frac{2}{7} \operatorname{Tr}_{K / \mathbb{Q}}\left(\alpha h\left(x_{2}, x_{3}\right)\right)-\frac{8}{7},
\end{aligned}
$$

and similarly

$$
\begin{aligned}
\left|\operatorname{Tr}_{K / \mathbb{Q}}\left(\frac{\alpha}{\sqrt{-7}} h\left(\bar{\alpha} x_{3}+x_{2}, x_{4}\right)\right)\right| \leq & h\left(x_{4}, x_{4}\right)+\frac{2}{7} h\left(\bar{\alpha} x_{3}+x_{2}, \bar{\alpha} x_{3}+x_{2}\right) \\
& -\mathrm{N}_{h}\left(\mathcal{O}_{K} x_{4} \oplus \mathcal{O}_{K} \frac{\alpha}{\sqrt{-7}}\left(\bar{\alpha} x_{3}+x_{2}\right)\right)
\end{aligned}
$$




$$
\begin{aligned}
\leq & h\left(x_{4}, x_{4}\right)+\frac{4}{7} h\left(x_{3}, x_{3}\right)+\frac{2}{7} h\left(x_{2}, x_{2}\right) \\
& +\frac{2}{7} \operatorname{Tr}_{K / \mathbb{Q}}\left(\bar{\alpha} h\left(x_{3}, x_{2}\right)\right)-\frac{8}{7} .
\end{aligned}
$$

From this it follows at once that

$$
h(\omega, \omega) \geq \frac{1}{7} h\left(x_{2}, x_{2}\right)+\frac{1}{7} h\left(x_{3}, x_{3}\right)+\frac{16}{7} \geq \frac{24}{7}>3,
$$

whence the conclusion.

REMARK. It can be checked easily that the arguments above would apply similarly to yield an extremal 36-dimensional 3-modular lattice (resp. an extremal 72-dimensional unimodular lattice) by tensoring over $\mathcal{O}_{K}$ a 9-dimensional (hermitian) unimodular $\mathcal{O}_{K}$-lattice of (hermitian) minimum 4 with $\mathbb{D}_{4}$ (resp. $\mathbb{E}_{8}$ ), as before. Unfortunately, it has been shown by Schiemann, using Kneser's neighbouring algorithm [Sc], that such a 9-dimensional unimodular $\mathcal{O}_{K}$-lattice, which would be extremal 18-dimensional 7-modular as a $\mathbb{Z}$-lattice, does not exist.

4.3. Barnes-Wall lattices. By a recursive application of tensor product, we construct two families of lattices, one of which can be identified a posteriori with the Barnes-Wall lattices.

Let $H=\mathbb{Q}_{2, \infty}$. We consider $\mathfrak{M}_{0}$ and $\mathfrak{P}$ as defined in Subsection 4.1. Viewed as $\mathbb{Z}[i]$-hermitian lattices, they are endowed with the hermitian form $h_{0}$, the matrix of which in the $\mathbb{Z}[i]$-basis $\{1, \omega=(-1+i+j+k) / 2\}$ of $\mathfrak{M}_{0}$ is

$$
\left(\begin{array}{cc}
1 & \frac{-1+i}{2} \\
\frac{-1-i}{2} & 1
\end{array}\right)
$$

As left (resp. right) $\mathfrak{M}_{0}$-lattices they are endowed with the hermitian form $h_{l}(x, y)=\bar{x} \cdot y\left(\operatorname{resp} . h_{r}(x, y)=\bar{y} \cdot x\right)$. This will be implicit, depending on the context, in the following. Next in the orthogonal direct sum of two copies of $\mathfrak{M}_{0}$ we consider the submodule $\{(x, y) \mid x \equiv y \bmod \mathfrak{P}\}$, which is isometric to $\mathbb{E}_{8}$ as a $\mathbb{Z}$-lattice (see [M, chapitre IV, §8]). In order to define the Barnes-Wall series we need the following lemma:

Lemma 4.3.1. (1) Let $(M, h)$ be a left $\mathbb{Z}[i]$-lattice. Then $\mathfrak{P} \otimes_{\mathbb{Z}[i]} M$ is canonically a left $\mathfrak{M}_{0}$-lattice for a suitable hermitian form over $\mathfrak{M}_{0}$.

(2) Let $(N, h)$ be a left $\mathfrak{M}_{0}$-lattice. Then $\mathbb{E}_{8} \otimes_{\mathfrak{M}_{0}} N$ is canonically a $\mathbb{Z}[i]$-lattice for a suitable hermitian form over $\mathbb{Z}[i]$.

P r o o f. (1) Under the identification $\mathfrak{M}_{0}=\mathbb{Z}[i] \oplus \mathbb{Z}[i] \omega$, we get a structure of $\left(\mathfrak{M}_{0}, \mathbb{Z}[i]\right)$-bimodule (left multiplication by $\mathfrak{M}_{0}$, right multiplication by $\mathbb{Z}[i])$, and consequently a well defined left- $\mathfrak{M}_{0}$-module structure on the tensor product $\mathfrak{P} \otimes_{\mathbb{Z}[i]} M$. It remains to prove that the inner product $\operatorname{Tr}_{\mathbb{Q}(i) / \mathbb{Q}}\left(h_{0} \otimes h\right)$ 
comes from an hermitian form $\widetilde{h}$ over $\mathfrak{M}_{0}$ on $\mathfrak{P} \otimes_{\mathbb{Z}[i]} M$, i.e.

$$
\operatorname{Tr}_{\mathbb{Q}(i) / \mathbb{Q}}\left(h_{0} \otimes h\right)=\operatorname{tr}(\widetilde{h}) .
$$

From [St, addendum to trace lemma 2.6], it amounts to checking that

$$
\begin{array}{r}
\operatorname{Tr}_{\mathbb{Q}(i) / \mathbb{Q}}\left(h_{0}\left(\alpha x_{1}, y_{1}\right) h\left(x_{2}, y_{2}\right)\right)=\operatorname{Tr}_{\mathbb{Q}(i) / \mathbb{Q}}\left(h_{0}\left(x_{1}, \bar{\alpha} y_{1}\right) h\left(x_{2}, y_{2}\right)\right), \\
\alpha \in \mathfrak{M}_{0}, x_{i} \in \mathfrak{P}, y_{i} \in M,
\end{array}
$$

which is satisfied since

$$
\operatorname{Tr}_{\mathbb{Q}(i) / \mathbb{Q}}\left(h_{0}\left(x_{1}, y_{1}\right) h\left(x_{2}, y_{2}\right)\right)=\operatorname{tr}\left(x_{1} \overline{y_{1} h\left(x_{2}, y_{2}\right)}\right),
$$

by definition of $h_{0}$.

(2) As before we see that $\mathbb{E}_{8}$ is a $\left(\mathbb{Z}[i], \mathfrak{M}_{0}\right)$-bimodule, and thus $\mathbb{E}_{8} \otimes_{\mathfrak{M}_{0}} N$ is a $\mathbb{Z}[i]$-module by left multiplication, and we just have to check that

$$
\begin{aligned}
\operatorname{tr}\left(h_{r}\left(\alpha x_{1}, y_{1}\right) h\left(x_{2}, y_{2}\right)\right)=\operatorname{tr}\left(h_{r}\left(x_{1}, \bar{\alpha} y_{1}\right) h\left(x_{2}, y_{2}\right)\right), & \\
& \alpha \in \mathbb{Z}[i], x_{i} \in \mathbb{E}_{8}, y_{i} \in N,
\end{aligned}
$$

which is obviously fulfilled.

We now define two infinite series: $L_{2}=\mathfrak{M}_{0}$ and, for $k \geq 1$,

$$
L_{2 k+1}=\mathbb{E}_{8} \otimes_{\mathfrak{M}_{0}} L_{2 k}, \quad L_{2 k+2}=\mathfrak{P} \otimes_{\mathbb{Z}[i]} L_{2 k+1}
$$

and similarly $M_{1}=\Lambda_{24}$ viewed as a $\mathfrak{M}_{0}$-lattice, and, for $k \geq 1$,

$$
M_{2 k+1}=\mathbb{E}_{8} \otimes_{\mathfrak{M}_{0}} M_{2 k}, \quad M_{2 k}=\mathfrak{P} \otimes_{\mathbb{Z}[i]} M_{2 k-1} .
$$

Proposition 4.3.2. (1) $L_{n}$ is a $2^{n}$-dimensional lattice with minimal norm $2^{\lfloor n / 2\rfloor}$, unimodular if $n$ is odd, 2-modular if $n$ is even.

(2) $M_{n}$ is a $12 \cdot 2^{n}$-dimensional lattice with minimal norm $2^{\lfloor n / 2\rfloor+2}$, unimodular if $n$ is odd, 2-modular if $n$ is even.

Pr o of. We only give the proof of the first assertion (the second is similar). Using the results of Section 2, one easily checks that $L_{n}^{*}=L_{n}$ for $n$ odd and $(1+i) L_{n}^{*}=L_{n}$ for $n$ even, whence the modularity. Then we can compute the minimal norm recursively, using the results of Section 3. Assuming first that $\mathrm{N}\left(L_{2 k}\right)=2^{k}$, let us prove that $\mathrm{N}\left(\mathbb{E}_{8} \otimes_{\mathfrak{M}_{0}} L_{2 k}\right)=\mathrm{N}\left(L_{2 k}\right)=2^{k}$. Since rank $\mathfrak{M}_{0} \mathbb{E}_{8}=2$, the rank of vectors in $\mathbb{E}_{8} \otimes_{\mathfrak{M}_{0}} L_{2 k}$ is at most 2. Since $\mathrm{N}_{h}\left(\mathbb{E}_{8}\right) \cdot \mathrm{N}_{h}\left(L_{2 k}\right)=2^{k-1}$, the hermitian norm of split vectors is at least $2^{k-1}$. For non-split vectors, we only need to check, using Proposition 3.2, that

$$
2 d_{2}\left(\mathbb{E}_{8}\right)^{1 / 2} d_{2}\left(L_{2 k}\right)^{1 / 2} \geq 2^{k-1} .
$$

Since $d_{2}\left(\mathbb{E}_{8}\right)=d_{\mathbb{E}_{8}}=1 / 2$ from $(9)$, it amounts to proving that

$$
d_{2}\left(L_{2 k}\right) \geq 2^{2 k-3}=\frac{1}{2} \mathrm{~N}_{h}\left(L_{2 k}\right)^{2} .
$$


Now $\mathfrak{M}_{0}$ is (right and left) euclidean, with euclidean minimum $1 / 2$, and a similar argument to the one we used in the proof of Lemma 4.2.2 gives the desired result.

If we now assume that $\mathrm{N}\left(L_{2 k+1}\right)=2^{k}$, we prove in the same way that $\mathrm{N}\left(\mathfrak{P} \otimes_{\mathbb{Z}[i]} L_{2 k+1}\right)=2^{k+1}$; this is clear for split vectors, and for non-split ones we just have to check that $d_{2}\left(L_{2 k+1}\right)^{2} \geq 2^{2 k-3}=\frac{1}{2} \mathrm{~N}_{h}\left(L_{2 k+1}\right)^{2}$, which is again clear since $\mathbb{Z}[i]$ has euclidean minimum $1 / 2$.

REMARK. One can prove that $L_{n}$ is isometric to the Barnes-Wall lattice $B W_{n}$ for any $n$. This amounts to an elementary (but tedious) identification, using E. S. Barnes and G. E. Wall's original paper [B-W]. As pointed out by the referee, the recursive construction of $B W_{n}$ is well known, and Proposition 4.3.2 is just a reformulation of the so-called construction E (see [C-S, Chapter 8]).

4.4. Concluding remarks. We conclude with examples which illustrate the fact that tensoring often fails to give dense lattices. This is related to the following lemma, which is the "hermitian" version of a result by R. Steinberg (see [M-H, Chapter 2, Theorem 9.6]):

LEMMA 4.4.1. Let $L$ be an $m$-dimensional hermitian $\mathcal{O}_{K}$-lattice, where $K$ is either an imaginary quadratic field or a quaternion field over $\mathbb{Q}$.

(1) $L^{\#} \otimes_{\mathcal{O}_{K}} L \simeq \operatorname{Hom}_{\mathcal{O}_{K}}(\bar{L}, \bar{L})$.

(2) The hermitian norm of $L^{\#} \otimes_{\mathcal{O}_{K}} L$ is at most $m$.

Proof. (1) First recall that if $(L, h)$ is a left hermitian lattice, then $(\bar{L}, \bar{h})$ is canonically a right hermitian lattice, where $\bar{L}=L$ as an additive group, and the right module structure is defined by $l \cdot \alpha=\bar{\alpha} l, l \in L, \alpha \in \mathcal{O}_{K}$. So the tensor product $L^{\#} \otimes L$ is well defined, even in the non-commutative case. The isomorphism is thus given by

$$
L^{\#} \otimes \mathcal{O}_{K} L \simeq \operatorname{Hom}_{\mathcal{O}_{K}}(\bar{L}, \bar{L}), \quad x \otimes y \mapsto z \mapsto h(z, x) y .
$$

(2) Under the above isomorphism, the identity element in $\operatorname{Hom}_{\mathcal{O}_{K}}(\bar{L}, \bar{L})$ corresponds to $\sum_{i=1}^{m} e_{i}^{\#} \otimes e_{i}$, where $\left\{e_{i}\right\}$ is any basis of $L$, and $\left\{e_{i}^{\#}\right\}$ its dual basis, and its hermitian norm is easily checked to be $m$.

Example 1. It is shown in $[\mathrm{H}$, Section 6$]$, that there is only one irreducible unimodular 3-dimensional hermitian lattice $L$ over $\mathbb{Z}[(1+\sqrt{-7}) / 2]$, with hermitian norm 2. So, from the previous lemma, $L \otimes_{\mathbb{Z}[(1+\sqrt{-7}) / 2]} L$ has hermitian norm at most 3 , and at least 3 from Proposition 3.2. Let $\omega=\sum_{i=1}^{3} x_{i} \otimes y_{i}$ be a minimal vector. The equality $h(\omega, \omega)=3$ implies that all inequalities in Proposition 3.2, for $r=3$, are in fact equalities. In other words, $\left\{x_{i}\right\}$ is a basis of $L$, and there is a unitary automorphism $\sigma$ of $L$ such that $y_{i}=\sigma\left(x_{i}^{\#}\right)$, where $\left\{x_{i}^{\#}\right\}$ denotes the dual basis of $\left\{x_{i}\right\}$. Moreover, one easily checks that the map which to any minimal vector $\sum_{i=1}^{3} x_{i} \otimes \sigma x_{i}^{\#}$ 
associates the unitary automorphism $\sigma$ is a well defined bijection between $S_{h}(L \otimes L)$ and the group $\operatorname{Aut}_{h}(L)$ of unitary automorphisms of $L$. As a $\mathbb{Z}$-lattice, $L \otimes L$ is thus a non-extremal (norm 6) 7-modular 18-dimensional lattice. This is the lattice denoted by $\left[ \pm\left(L_{2}(7) \otimes_{\sqrt{-7}}^{2} L_{2}(7)\right) \cdot 2\right]_{18}$ in $[\mathrm{N}-\mathrm{P}$, p. 44], and the number of minimal vectors which is given there is in accordance with $\left|\operatorname{Aut}_{h}(L)\right|=336$ as mentioned in $[\mathrm{H}]$.

ExAMPLE 2. Let $L_{1}$ and $L_{2}$ be two hermitian unimodular lattices of rank 3 over an (essentially unique) maximal order $\mathfrak{M}$ in the quaternion field $\mathbb{Q}_{3, \infty}$, of minimal hermitian norm 2 (it is known that at least one such lattice exists, namely the Coxeter-Todd lattice $K_{12}$, see for instance [B, Theorem 6.6]). The same argument as above leads to the following alternative: either $L_{1}$ and $L_{2}$ are isometric as hermitian lattices, whence $\bar{L}_{1} \otimes_{\mathfrak{M}} L_{2}$ has hermitian norm 3 (the minimal vectors being in one-to-one correspondence with the hermitian isometries from $L_{1}$ onto $L_{2}$ ), or they are not, whence $\bar{L}_{1} \otimes_{\mathfrak{M}} L_{2}$ has hermitian norm 4 , and, as a $\mathbb{Z}$-lattice, is an extremal 3-modular 36dimensional lattice. Unfortunately, one can check (see the proof of Theorem 6.6 in $[\mathrm{B}]$ ) that up to isometry there is only one hermitian unimodular lattice of rank 3 over $\mathfrak{M}$.

REMARK. For the same reason, the construction $\Lambda_{24} \otimes_{\mathfrak{M}} K_{12}$, where $\Lambda_{24}$ is the Leech lattice, fails to give an extremal unimodular 72-dimensional lattice. So the classification in low dimensions tends to confirm the widely held opinion that extremal lattices of level respectively $1,3,7$ and dimension $72,36,18$ do not exist.

\section{References}

[ATLAS] J. H. Conway, R. T. Curtis, S. P. Norton, R. A. Parker and R. A. Wilson, Atlas of Finite Groups, Oxford Univ. Press, Oxford, 1985.

[B] C. Bachoc, Applications of coding theory to the construction of modular lattices, J. Combin. Theory Ser. A 78 (1997), 92-119.

[B-N] C. Bachoc and G. Nebe, Extremal lattices of minimum 8 related to the Mathieu group $M_{22}$, J. Reine Angew. Math., to appear.

[B-W] E. S. B arnes and G. E. W all, Some extreme forms defined in terms of Abelian groups, J. Austral. Math. Soc. 1 (1959), 47-63.

[Bou] N. Bourbaki, Algèbre, Hermann, Paris, 1970.

[C-S] J. H. Conway and N. J. A. Sloane, Sphere Packings, Lattices and Groups, 3rd ed., Grundlehren Math. Wiss. 290, Springer, New York, 1999.

[H] D. W. Hoffmann, On positive definite Hermitian forms, Manuscripta Math. 71 (1991), 399-429.

[K] Y. Kitaoka, Arithmetic of Quadratic Forms, Cambridge Univ. Press, 1993.

[K1] _, Scalar extension of quadratic lattices II, Nagoya Math. J. 67 (1977), 159164. 
[M] J. Martinet, Les Réseaux Parfaits des Espaces Euclidiens, Masson, Paris, 1996.

[M-H] J. Milnor and J. D. Husemoller, Symmetric Bilinear Forms, Ergeb. Math. Grenzgeb. 73, Springer, New York, 1973.

[N-P] G. Nebe and W. Plesken, Finite rational matrix groups, Mem. Amer. Math. Soc. 556 (1995).

[Q] H.-G. Quebbemann, Modular lattices in euclidean spaces, J. Number Theory 54 (1995), 190-202.

[R] I. Reiner, Maximal Orders, Academic Press, London, 1975.

[Sc] A. Schiemann, private communication.

[Se] J.-P. Serre, Corps Locaux, Hermann, Paris, 1968.

[St] N. W. Stoltzfus, Unraveling the knot concordance group, Mem. Amer. Math. Soc. 192 (1977).

Université Bordeaux I

Mathématiques

351, cours de la Libération

33405 Talence Cedex, France

E-mail: coulange@math.u-bordeaux.fr

Received on 16.3.1998

and in revised form on 22.9.1999 